\title{
Variaciones dialectales del castellano y distancias comunicacionales en estudiantes universitarios. Un estudio sobre habilidades psicolingüísticas y bilingüismo en el Perú
}

Changes in the castilian dialect and communication distances in university students. A study of psycholinguistic skills and bilingualism in Peru

Ricardo Canales G. ; Esther Velarde C.; Magali Meléndez J.; Susana lingán H.

Universidad Nacional Mayor de San Marcos, Lima, Perú

(RECIBIDO 20/06/2015, AcEPTADO 10/10/2015)

\section{RESUMEN}

El estudio buscó establecer si existirían diferencias psicolingüísticas y comunicacionales en estudiantes universitarios, considerando su diversa realidad cultural y sociolingüística. Se examinó a 20 alumnos de la Universidad Nacional Mayor de San Marcos (dialecto del castellano ribereño costeño), 18 alumnos de la Universidad Nacional San Agustín de Arequipa (castellano sur andino) y 28 alumnos de la Universidad Nacional de Huancavelica (condición interlectal quechua-castellano).

Se empleó el Test breve de bilingüismo (González 2006) y Pruebas psicolingüísticas experimentales: percepción auditiva, procesos léxicos, procesos semánticos y procesos pragmáticos, tanto en la comprensión como en la producción oral.

Los resultados mostraron diferencias en la mayoría de dimensiones del lenguaje oral: comprensión (percepción del habla, reconocimiento léxico, dominio sintáctico y semántico) y producción (reconocimiento de objetos, fluidez semántica, dominio sintáctico y pragmático) a favor del grupo de Lima en desmedro de los estudiantes principalmente de Huancavelica (condición interlectal quechua-castellano). Dicha diferencia en procesos psicolingüísticos, se manifiesta en un déficit en los procesos comunicacionales.

Palabras clave: Dialectos del español, habilidades psicolingüísticas, estudiantes universitarios, bilingüismo.

\section{ABSTRACT}

The study sought to determine if differences exist psycholinguistic and communication among university students, given their different linguistic and sociocultural reality. 20 students of Greater National University of San Marcos (Castilian dialect of coastal riverine), 18 students of the National University San Agustin de Arequipa (Castilian southern Andes) and 28 students of the National University of Huancavelica (Quechua Castilian interlectal condition) was examined ).

1 Docente Auxiliar de la Facultad de Psicología de la UNMSM, E-mail: ricardo.is.canales@gmail.com 
VARIACIONES DIALECTALES DEL CASTELLANO Y DISTANCIAS COMUNICACIONALES EN ESTUDIANTES UNIVERSITARIOS...

The brief bilingual Test (González 2006) and experimental psycholinguistic tests was used: auditory perception, lexical processes, semantic and pragmatic processes, both in understanding and oral production.

The results showed differences in most dimensions of oral language: comprehension (speech perception, word recognition, syntactic and semantic domain) and production (object recognition, semantic fluency, syntactic and pragmatic domain) for the group at the expense of Lima students mainly from Huancavelica (Quechua Castilian interlectal condition). This difference in psycholinguistic processes, manifests itself in a deficit in communication processes.

Keywords: Spanish dialects, psycholinguistic skills, university students, bilingualism.

\section{INTRODUCCIÓN}

Los últimos resultados de la Prueba PISA (2012) en la que nuestros alumnos de 15 años de edad, ocupan el último lugar en competencias lectoras, y también en matemáticas y ciencias, entre 65 países del mundo -por debajo de Albania, Túnez o Qatar-, ha dado mucho que hablar. Incluso, comparando con rendimientos de años anteriores, el drama educativo nacional se ha profundizado. Y la pregunta principal es: ¿cuál es la razón o explicación de todo ello?

$\mathrm{Al}$ respecto, poco se ha incidido en el hecho que los rendimientos educativos y logros de aprendizaje de los niños en el Perú, difieren radicalmente entre lo que alcanzan los alumnos de zonas urbanas y lo que logran los de zonas rurales. Y lo cierto es que al paso de los años -y muy a pesar de que se hable del crecimiento económico en el Perú- dicha brecha va en aumento.

Los niños del Perú atraviesan una serie de dificultades en su proceso de desarrollo psíquico: cognitivo, afectivo, psicolingüístico, social. Existen una serie de factores socioculturales que intervienen negativamente, afectando dicho desarrollo. Entre ellos tenemos a la pobreza extrema, la marginalidad social y cultural, el analfabetismo de los padres, el bilingüismo (Gonzáles, 2006). Cuando los niños llegan a la escuela, traen una serie de dificultades, lo cual no les permite formar y adquirir las competencias básicas -indispensables para insertarse en el mundo moderno-: lectura, escritura, matemáticas, ciencias. Y el lenguaje es una habilidad fundamental para la exitosa realización de esos logros. En un estudio (Canales, 2012), se encontró que a nivel de conciencia fonémica, conocimiento del nombre y sonido de las letras, los bilingües incipientes rendían más bajo que los niños monolingües castellano y bilingües avanzados. En otro estudio (Canales, 2013) examinando el desarrollo del lenguaje oral y las habilidades prelectoras (conciencia fonológica) en niños de 4 a 6 años, se encontró que los niños de la comunidad de Quintaojos (Huancavelica) en donde predominan los bilingües incipientes, obtenían los más bajos rendimientos, en comparación a los niños de Huayobamba (Cajamarca) que son monolingües españoles y los niños de Lima: Cercado y Ate-Vitarte.

En particular, en el presente estudio, se quería ver que es lo que ocurre a nivel psicolingüístico con alumnos universitarios de la carrera de educación -considerando que en sus manos estarán los alumnos en los próximos años cuando se gradúen 
de maestros- pertenecientes a diversas realidades socioculturales y lingüísticas. González (2006) ha incidido en el grave problema comunicacional de los peruanos, cuando en sus estudios sobre las variedades dialectales del español, siguiendo a Escobar (1976, 1978), encontró que ante tareas de carácter psicolingüístico (percepción auditiva, memoria verbal, asociaciones verbales, hábitos verbales), los más deficientes, precisamente resultaban los bilingües.

Por lo tanto, en el presente estudio se buscó analizar si existen diferencias en habilidades psicolingüísticas y comunicacionales en estudiantes universitarios de educación considerando las diversas formas dialectales del español en el Perú. Se estudió si a esta diversidad sociolingüística le correspondía una diversa realidad psicolingüística y siendo así, de qué forma se afectarían sus procesos comunicacionales.

\section{Objetivos}

- Examinar las variables psicolingüísticas: percepción auditiva, memoria verbal, asociaciones verbales, hábitos verbales, en Los alumnos con dialecto ribereño costeño, dialecto sur-andino, y de condición interlectal quechua-castellano.

- Determinar si existen diferencias en dichas habilidades psicolingüísticas entre los alumnos de los grupos examinados.

- Establecer los grados y jerarquías en el procesamiento de la información que proviene en "norma culta limeña" entre los grupos examinados.

\section{Hipótesis}

- Existen diferencias en el nivel de desarrollo de las habilidades psicolingüísticas en los alumnos universitarios en función a su realidad sociolingüística.

- Existirá una gradiente en términos de la eficacia en los procesos psicolingüísticos a favor de los hablantes del castellano ribereño costeño y en desmedro de los hablantes condición interlectal quechua- castellano.

\section{MÉTODO}

El presente fue un estudio descriptivo, de tipo causal comparativo, en donde diferenciando a los estudiantes universitarios por su condición sociolingüística, se trató de ver como se han desarrollado las variables psicolingüísticas: : percepción auditiva, memoria verbal, asociaciones verbales, hábitos verbales.

Se controlaron las variables: edad, sexo y condición sociolingüística.

$\mathrm{Al}$ final se trató de observar las diferencias que pueden aparecer entre los grupos examinados a nivel de las variables psicolingüísticas.

Asimismo, sobre esa base se estableció la jerarquía o gradiente entre los grupos 
VARIACIONES DIALECTALES DEL CASTELLANO Y DISTANCIAS COMUNICACIONALES EN ESTUDIANTES UNIVERSITARIOS...

respecto a dichas variables y su eficacia para el tratamiento de la información que proviene en "norma culta limeña".

La población estuvo constituida por alumnos que ingresan a estudiar la carrera de educación: alumnos con dialecto ribereño costeño: Universidad Nacional Mayor de San Marcos; dialecto sur-andino: Universidad San Agustín de Arequipa; condición interlectal quechua-castellano: alumnos de la Universidad Nacional de Huancavelica.

La muestra se escogió de modo intencional, considerando la posibilidad de acceso a dichos estudiantes, especialmente pensando en los de provincias.

\section{Técnicas e instrumentos de recolección de datos:}

Se aplicaron los siguientes instrumentos:

1) Test breve de bilingüismo quechua-castellano de Escobar, en adaptación de González.

2) Pruebas psicolingüísticas experimentales, expresamente diseñadas para examinar: percepción auditiva, procesos léxicos, procesos semánticos y procesos pragmáticos, tanto en la comprensión como en la producción oral.

\section{RESULTADOS \\ HIPÓTESIS:}

Existen diferencias en el nivel de desarrollo de las habilidades psicolingüísticas en los alumnos universitarios en función a su realidad sociolingüística.

En la tabla 1 se puede apreciar que existen diferencias significativas en la mayoría de dimensiones del lenguaje oral, con excepción de las tareas vinculadas con la función pragmática del lenguaje. En estas tareas, incluso, los estudiantes de Huancavelica presentaron un mejor desempeño que sus pares en Lima y Arequipa.

De acuerdo con los análisis post hoc, en las tareas de Percepción del habla, Reconocimiento de errores gramaticales, Nominación de objetos y acciones, Fluidez semántica y Construcción correcta de oraciones; el grupo de Huancavelica no se diferencia del grupo de Arequipa, pero ambos se diferencias del grupo de Lima.

En las tareas de Reconocimiento de Palabras y de Evaluación general de la producción oral, el grupo de Huancavelica se diferencia tanto del grupo de Arequipa y del de Lima, pero estos no se diferencian entre sí. En la Comprensión de textos, solo se encuentran diferencias entre el grupo de Huancavelica y el grupo de Lima. En la tarea de Función Pragmática (I nivel) solo se encuentran diferencias entre Huancavelica y Arequipa, a favor de Huancavelica.

En un análisis discriminante exploratorio, se encuentra que las tareas que podrían discriminan más el desempeño de los tres grupos son: Reconocimiento de errores 
gramaticales, Nominación de objetos y acciones y Evaluación general del lenguaje productivo.

Tabla 1. Estadísticos y dimensiones del lenguaje oral.

\begin{tabular}{|c|c|c|c|c|c|c|}
\hline \multicolumn{2}{|c|}{ Dimensiones del lenguaje oral } & \multirow{2}{*}{$\begin{array}{l}\mathrm{N} \\
28\end{array}$} & \multirow{2}{*}{$\begin{array}{l}\text { Media } \\
25,286\end{array}$} & \multirow{2}{*}{$\begin{array}{c}\begin{array}{c}\text { Desviación } \\
\text { estándar }\end{array} \\
3,4839\end{array}$} & \multirow{2}{*}{$\begin{array}{c}\mathrm{F} \\
10,116\end{array}$} & \multirow{2}{*}{$\begin{array}{l}\text { Sig. } \\
, 000\end{array}$} \\
\hline \multirow{4}{*}{ Percepción del habla } & Huancavelica & & & & & \\
\hline & Arequipa & 18 & 26,500 & 2,6624 & & \\
\hline & Lima & 20 & 28,900 & 1,2524 & & \\
\hline & Total & 66 & 26,712 & 3,1170 & & \\
\hline \multirow{4}{*}{$\begin{array}{l}\text { Reconocimiento de } \\
\text { palabras correctas e } \\
\text { incorrectas }\end{array}$} & Huancavelica & 28 & 8,107 & 3,2127 & 14,087 & , 000 \\
\hline & Arequipa & 18 & 10,778 & 2,1298 & & \\
\hline & Lima & 20 & 12,100 & 2,1740 & & \\
\hline & Total & 66 & 10,045 & 3,1498 & & \\
\hline \multirow{4}{*}{$\begin{array}{l}\text { Reconocimiento de } \\
\text { errores gramaticales }\end{array}$} & Huancavelica & 28 & 10,571 & 2,0804 & 51,881 & 000 \\
\hline & Arequipa & 18 & 12,389 & 1,2897 & & \\
\hline & Lima & 20 & 15,750 & 1,5517 & & \\
\hline & Total & 66 & 12,636 & 2,7881 & & \\
\hline \multirow{4}{*}{$\begin{array}{l}\text { Asociación de } \\
\text { oraciones con dibujos }\end{array}$} & Huancavelica & 28 & 3,071 & 1,0516 & 11,562 & 000 \\
\hline & Arequipa & 18 & 3,556 & 1,4234 & & \\
\hline & Lima & 20 & 4,600 &, 7539 & & \\
\hline & Total & 66 & 3,667 & 1,2568 & & \\
\hline \multirow{4}{*}{$\begin{array}{l}\text { Comprensión de } \\
\text { textos }\end{array}$} & Huancavelica & 28 & 2,679 & 1,2781 & 6,233 & ,003 \\
\hline & Arequipa & 18 & 3,111 & 1,2314 & & \\
\hline & Lima & 20 & 4,000 & 1,3377 & & \\
\hline & Total & 66 & 3,197 & 1,3836 & & \\
\hline \multirow{4}{*}{$\begin{array}{l}\text { Nominación de } \\
\text { objetos y acciones }\end{array}$} & Huancavelica & 28 & 6,500 & 4,5583 & 48,832 & , 000 \\
\hline & Arequipa & 18 & 9,389 & 5,3373 & & \\
\hline & Lima & 20 & 20,500 & 5,1453 & & \\
\hline & Total & 66 & 11,530 & 7,7939 & & \\
\hline \multirow{4}{*}{ Fluidez semántica } & Huancavelica & 28 & 33,679 & 7,7558 & 14,265 & 000 \\
\hline & Arequipa & 18 & 39,889 & 11,5192 & & \\
\hline & Lima & 20 & 50,200 & 12,9070 & & \\
\hline & Total & 66 & 40,379 & 12,5487 & & \\
\hline \multirow{4}{*}{$\begin{array}{l}\text { Construcción } \\
\text { correcta de oraciones }\end{array}$} & Huancavelica & 28 & 8,357 & 2,4527 & 10,794 &, 000 \\
\hline & Arequipa & 18 & 7,722 & 2,3962 & & \\
\hline & Lima & 20 & 10,900 & 1,9167 & & \\
\hline & Total & 66 & 8,955 & 2,6102 & & \\
\hline \multirow{4}{*}{$\begin{array}{l}\text { Funciones } \\
\text { pragmáticas }\end{array}$} & Huancavelica & 28 & 4,393 &, 7373 & 3,648 &, 032 \\
\hline & Arequipa & 18 & 3,500 & 1,6179 & & \\
\hline & Lima & 20 & 4,200 & 1,0052 & & \\
\hline & Total & 66 & 4,091 & 1,1599 & & \\
\hline
\end{tabular}


VARIACIONES DIALECTALES DEL CASTELLANO Y DISTANCIAS COMUNICACIONALES EN ESTUDIANTES UNIVERSITARIOS...

\begin{tabular}{|c|c|c|c|c|c|c|}
\hline \multirow{4}{*}{$\begin{array}{l}\text { Organización del } \\
\text { discurso }\end{array}$} & Huancavelica & 28 & 3,857 & 1,5567 & 1,256 & 292 \\
\hline & Arequipa & 18 & 4,722 & 2,4448 & & \\
\hline & Lima & 20 & 4,500 & 1,9331 & & \\
\hline & Total & 66 & 4,288 & 1,9515 & & \\
\hline \multirow{4}{*}{ Reglas sociales } & Huancavelica & 28 & 3,750 & ,8872 & ,430 & ,652 \\
\hline & Arequipa & 18 & 3,444 & 1,3382 & & \\
\hline & Lima & 20 & 3,750 & 1,4096 & & \\
\hline & Total & 66 & 3,667 & 1,1810 & & \\
\hline \multirow{4}{*}{$\begin{array}{l}\text { Situaciones } \\
\text { conversacionales }\end{array}$} & Huancavelica & 28 & 7,000 & 2,0548 & ,981 & ,381 \\
\hline & Arequipa & 18 & 6,389 & 2,5927 & & \\
\hline & Lima & 20 & 7,350 & 1,7554 & & \\
\hline & Total & 66 & 6,939 & 2,1331 & & \\
\hline \multirow{4}{*}{$\begin{array}{l}\text { Evaluación integral } \\
\text { de la producción } \\
\text { oral: funciones } \\
\text { pragmáticas de alto } \\
\text { nivel }\end{array}$} & Huancavelica & 28 & 10,071 & 3,2080 & 18,565 &, 000 \\
\hline & Arequipa & 17 & 13,235 & 2,5867 & & \\
\hline & Lima & 20 & 15,150 & 2,7198 & & \\
\hline & Total & 65 & 12,462 & 3,6232 & & \\
\hline
\end{tabular}

\section{HIPÓTESIS:}

Existirá una gradiente en términos de la eficacia en los procesos psicolingüísticos a favor de los hablantes del castellano ribereño costeño (Lima) y en desmedro de los hablantes de condición interlectal quechua-castellano.

Se logró establecer que efectivamente existe una gradiente en términos de uso y puesta en marcha de procesos psicolingüísticos que permiten un mejor procesamiento y asimilación de la información verbal que viene en lengua castellana a favor del grupo que emplea el castellano ribereño- costeño, en este caso los alumnos de la Universidad Nacional Mayor de San Marcos, Lima. Y los que tendrían más afectados dichos procesos son los alumnos de condición interlectal quechua- castellano, de la Universidad Nacional de Huancavelica. La excepción en este punto es lo que ocurre con las funciones pragmáticas del lenguaje, donde estos rinden igual que los de Lima, y que en el punto de Discusión analizaremos.

A continuación, se presentan las figuras de la distribución de medias para una mayor apreciación de las diferencias de los grupos. En dichos gráficos estadísticos, la coordenada $\mathrm{X}$ expresa los puntajes alcanzados en las diversas pruebas psicolingüísticas: Percepción del habla, Reconocimiento de palabras correctas e incorrectas, Reconocimiento de errores gramaticales, Asociación de oraciones con dibujos, Comprensión de textos, Nominación de objetos y acciones, Fluidez semántica, Construcción correcta de oraciones y Función pragmática, todas ellas representadas por sus iniciales; mientras que la coordenada Y, representa a los alumnos, de acuerdo a su ciudad: 1.0 Huancavelica, 2.0 Arequipa, 3.0 Lima. 
Ricardo Canales G.; Esther Velarde C.; Magali Meléndez J.; Susana Lingán H.
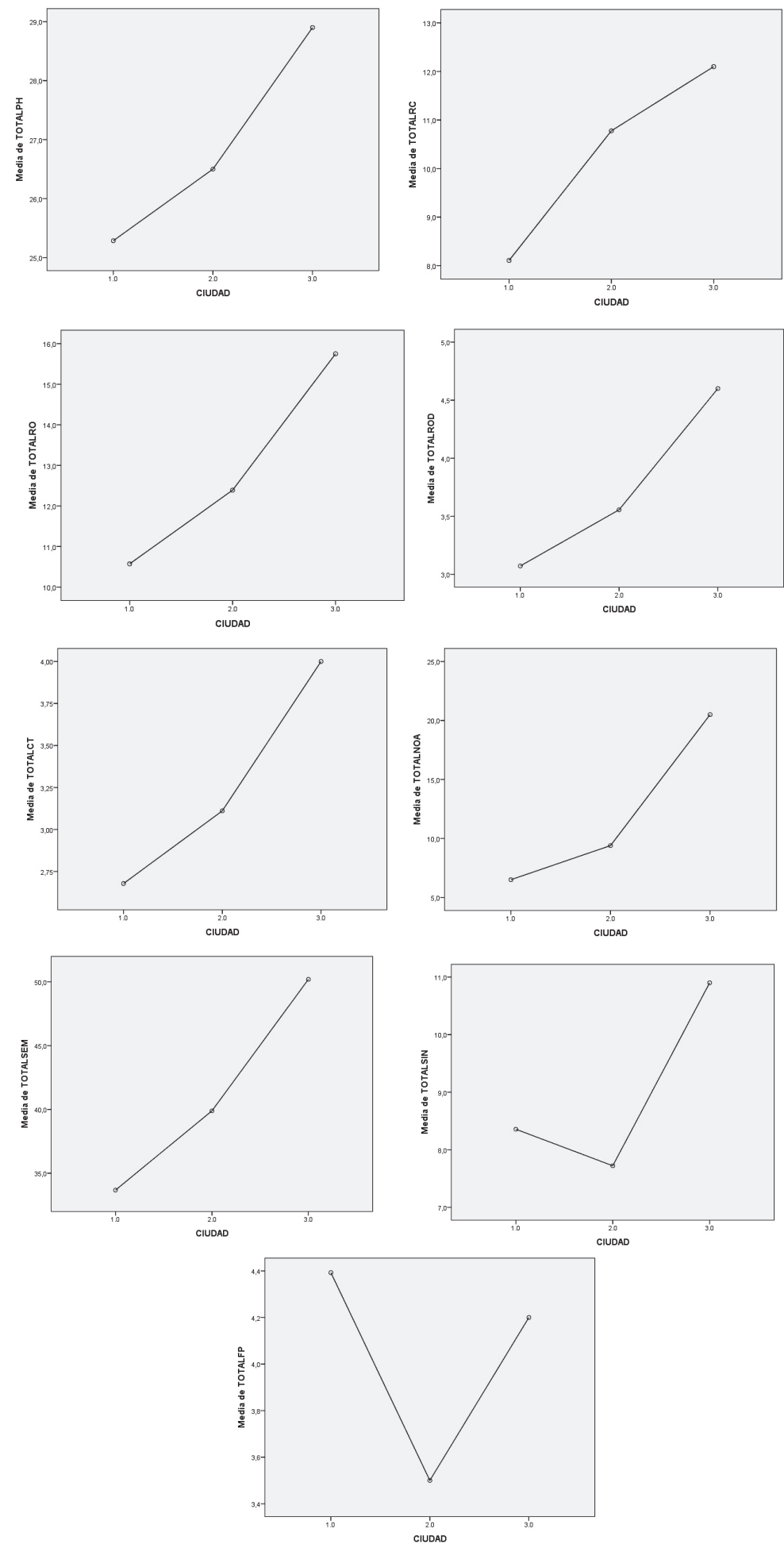

Revista de InVestigación en Psicología - Vol. 18, N. ${ }^{\circ} 2$ 
Al final presentamos la medida total en términos de media, para los 3 grupos: Huancavelica, Arequipa y Lima.

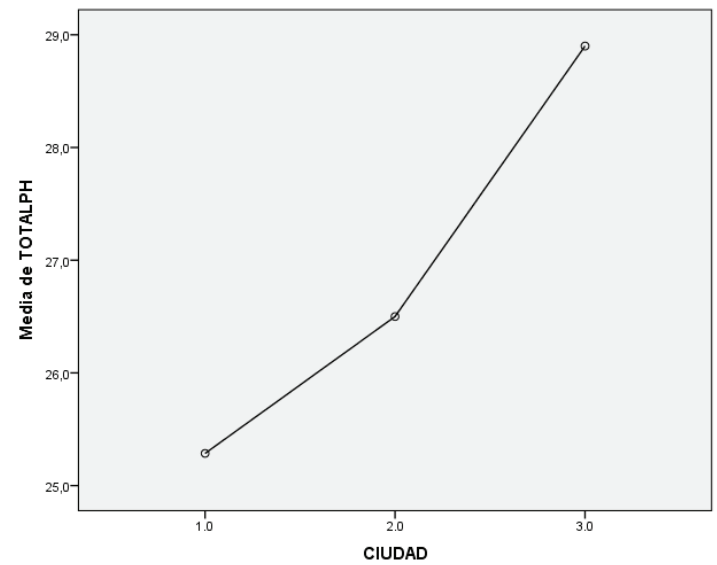

Figura 1. Distribución de medias de los estudiantes de Huancavelica (1), Arequipa (2) y Lima (3).

\section{DISCUSIÓN}

En el resultado principal del presente estudio apreciamos las importantes diferencias que aparecen en términos de habilidades psicolingüísticas entre los alumnos universitarios conformantes de los tres grupos, esto quiere decir que en tareas de percepción auditiva, procesos léxicos, procesos semánticos y procesos pragmáticos, así como en la comprensión y producción oral, los alumnos de Lima, rinden mejor que los de Arequipa y Huancavelica, y a su vez, los de Arequipa mejor que los de Huancavelica, en casi el conjunto de las tareas excepto en funciones pragmáticas.

¿Qué estaría indicando dicho resultado?

Indicaría que de todos modos ejercen una influencia en el desarrollo psicolingüístico, y en el procesamiento de la información verbal vertida en castellano ("norma culta limeña"), la marginalidad, la pobreza (propia del interior del país), aunado a la condición interlectal quechua-castellano (más presente en la muestra de Huancavelica, en donde la mayoría de los estudiantes tienen padres quechua-hablantes). La importancia de dichos factores disortogénicos había sido señalada por González (2006) y ratificada por Canales $(2012,2013)$.

Los alumnos evaluados (particularmente en Huancavelica), si bien no asumen la condición expresa de bilingües (incipientes o avanzados) si pueden caracterizarse como poseedores y hablantes del Castellano andino, el cual poseería características fonológicas, léxicas, sintácticas y semánticas que lo hacen distinto a las otras lenguas, así como a la norma culta limeña.

Se afirma que el castellano andino es una variedad lingüística que surge como producto de la fusión del castellano y una lengua andina, que puede ser hablado por monolingües del castellano en la sierra del Perú, constituyéndose en lengua 
materna de algunas regiones (Andrade, 2010). Escobar (1978), señalaba que existían diferencias en aspectos como: imprecisión en el vocalismo, tratamiento de los grupos vocálicos, imprecisión acentual, firmeza del consonantismo, interpolación de voces de la lengua materna o combinación de morfemas.

De otro lado con respecto al funcionamiento en la función pragmática en los estudiantes de Huancavelica, dentro de niveles similares a los de Arequipa, e incluso Lima, habría que señalar que en dicha tarea, se examinó aspectos comunicacionales muy elementales: p.e. se mostraba imágenes de una niña que observa a un pajarito muerto, y se le preguntaba al sujeto: ¿cómo está la niña?, ¿qué le ha pasado?; o en un nivel siguiente, se observa a un hombre vestido con los colores de su equipo favorito en el estadio, molesto, y se pregunta: ¿cómo está el señor?, ¿dónde se encuentra?, ¿quién va ganando el partido?.

En este tipo de tareas, los alumnos evaluados respondían dentro de lo esperado. Se entiende que para dar respuesta a dichas preguntas no se requiere mayores niveles de elaboración: se observa la imagen, se apelan a los recursos léxicos disponibles, y se expresa una respuesta. El problema viene cuando se le plantean situaciones más abstractas y de mayor complejidad, como cuando en nuestras pruebas experimentales psicolingüísticas, tratando de examinar las funciones pragmáticas de alto nivel, bajo el título de "Evaluación integral de la producción oral”, se le hace preguntas del tipo: ¿Por qué es importante que un país viva en democracia?, y ahí, si se vuelven a observar diferencias a favor de la muestra de Lima, y un rendimiento significativamente menor de la muestra de Huancavelica.

Respecto a nuestra segunda hipótesis, efectivamente se encontró que en términos de eficacia en los procesos psicolingüísticos y en el procesamiento de la información vertida en castellano, los alumnos de Lima rendían mejor que los de provincias, quedando los de Huancavelica (condición interlectal quechuacastellano) en último lugar, se puede afirmar, concordante con lo descubierto por González (2006) que son los grupos que presentan condición bilingüe o interlectal, quienes pierden una mayor cantidad de información, y pierden menos los que se encuentran en zonas de mayor interacción social y cultural, como es el caso de los estudiantes limeños.

Por lo demás, hay que indicar que está probado que un deficiente desarrollo de las habilidades psicolingüísticas y competencias adecuadas para procesar la información proveniente en castellano (norma culta), afecta la asimilación de los contenidos académico-científicos a los cuales el alumno está expuesto en la Universidad, redundando todo ello en la calidad de su formación profesional (Fregoso 2005).

\section{CONCLUSIONES}

1) Se hallaron diferencias significativas en el conjunto de las puntuaciones que examinaban dimensiones del lenguaje oral: comprensión (percepción del habla, reconocimiento léxico, dominio sintáctico y semántico) y producción 
VARIACIONES DIALECTALES DEL CASTELLANO Y DISTANCIAS COMUNICACIONALES EN ESTUDIANTES UNIVERSITARIOS...

(reconocimiento de objetos, fluidez semántica, dominio sintáctico y pragmático de alto nivel) a favor del grupo de Lima (castellano ribereño costeño) en desmedro de los estudiantes principalmente de Huancavelica (condición interlectal quechua- castellano).

2) En las tareas de Reconocimiento de Palabras, los grupos de Lima y Arequipa no se diferencian entre sí, aunque sí de modo significativo con el grupo de Huancavelica. En dominio semántico: Comprensión de textos, solo se encuentran diferencias entre el grupo de Huancavelica y el grupo de Lima, mas no entre Huancavelica y Arequipa.

3) Las tareas que discriminan más el desempeño de los tres grupos son: Reconocimiento de errores gramaticales, Nominación de objetos y acciones y Dominio pragmático (alto nivel).

4) Los instrumentos (pruebas, técnicas y procedimientos experimentales) expresamente diseñados para este estudio y utilizados en el examen de las variables psicolingüísticas mostraron su valor y utilidad.

5) Respecto a los aspectos comunicacionales y de procesamiento de la información verbal vertida en castellano, es el grupo de condición interlectal quechuacastellano: Huancavelica, el que se ve más afectado, marcándose en tal sentido una distancia comunicacional mayor en relación a los otros grupos.

\section{REFERENCIAS BIBLIOGRÁFICAS}

Andrade, L. (2010). Contactos y fronteras de lenguas en la Cajamarca prehispánica. Boletín de Arqueología PUCP, 14, 165-180.

Canales, R. (2014) “Diferencias en procesos y habilidades psicolingüísticas en niños monolingües castellano y bilingües quechua-castellano. Estudio de procesos psicolingüísticos y lectura en niños de $1^{\text {er }}$ grado". Lima. Theorema. Vol. 1. N. ${ }^{\circ}$ 1. Segunda época. UNMSM.

Canales, R. (2014) “ Diferencias en el desarrollo del lenguaje oral y en habilidades pre lectoras en niños de 4 a 6 años en condición de marginalidad y no marginalidad. Un estudio sobre marginalidad, cultura y bilingüismo en el Perú". Lima. Revista de Investigación en Psicología. Vol. 17. N¹. UNMSM.

Fregoso, G. (2005) Los problemas del estudiante universitario con la lectura. Un estudio de caso. Revista Universidad EAFIT Vol. 44. N. ${ }^{\circ}$ 149. 2008, pp. 9-22. Universidad de Guadalajara.

Gonzáles, R. (1995): Psicología del niño peruano. Lima: Universidad de Lima. Fac. de Psicología.

Gonzáles, R. (2006) Problemas psicológicos en la comunicación lingüística en el Perú. En: Problemas psicolingüisticos en el Perú. Lima. Edic. N. Reátegui. 
Ricardo Canales G.; Esther Velarde C.; Magali Meléndez J.; Susana Lingán H.

Gonzáles, R. (2008) Test breve de bilingüismo quechua- español TBB. En: Instrumentos psicolingüisticos. Lima. Edic. N. Reátegui.

Escobar, A. (1978) Variaciones sociolingüísticas del castellano en el Perú. Lima. IEP.

Pozzi-Escot, I. (1984) El castellano como segunda lengua en el Perú. Lima. Cielo Abierto. 
